\title{
Existence of bounded discrete steady state solutions of the van Roosbroeck system with monotone Fermi-Dirac statistic functions
}

\author{
K. Gärtner ${ }^{1,2}$
}

Published online: 16 June 2015

(C) Springer Science+Business Media New York 2015

\begin{abstract}
If in the classic van Roosbroeck system (Bell Syst Tech J 29:560-607, 1950) the statistic function is modified, the equations can be derived by a variational formulation or just using a generalized Einstein relation. In both cases a dissipative generalization of the ScharfetterGummel scheme (IEEE Trans Electr Dev 16, 64-77, 1969), understood as a one-dimensional constant current approximation, is derived for strictly monotone coefficient functions in the elliptic operator $\nabla \cdot f(v) \nabla, v$ chemical potential, while the hole density is defined by $p=\mathcal{F}(v) \leq e^{v}$. A closed form integration of the governing equation would simplify the practical use, but mean value theorem based results are sufficient to prove existence of bounded discrete steady state solutions on any boundary conforming Delaunay grid. These results hold for any piecewise, continuous, and monotone approximation of $f(v)$ and $\mathcal{F}(v)$. Hence an implementation based on this discretization will inherit the same stability properties as the Boltzmann case based on the ScharfetterGummel scheme. Large chemical potentials and and related degeneracy effects in semiconductors can be approximated. A proven, stability focused blueprint for the discretization of a fairly general, steady state Fermi-Dirac like drift-diffusion setting for semiconductors using mainly new results to extend classic ideas is the main goal.
\end{abstract}

Keywords Generalized Scharfetter-Gummel scheme . Fermi-Dirac statistics · Generalized Einstein relation . Dissipativity - Bounded discrete steady state solutions .

K. Gärtner

klaus.gaertner@usi.ch

1 Weierstrass Institute for Applied Analysis and Stochastics, Mohrenstr. 39, 10117 Berlin, Germany

2 Present Address: ICS, Universitá della Svizzera italiana, Via Giuseppe Buffi 13, 6904 Lugano, Switzerland
Unique thermodynamic equilibrium · Degenerate semiconductors

Mathematics Subject Classification $65 \mathrm{~N} 08 \cdot 65 \mathrm{~N} 12$. $35 \mathrm{~J} 55$

\section{Introduction}

In section two a short summary of the variational formulation of the drift-diffusion equations is given to illustrate its consequences and to compare it with the phenomenologic derivation based on the generalized Einstein relation, which holds at equilibrium. The analytic equations and boundary conditions are stated. Section three discusses the generalization of the Scharfetter-Gummel scheme as special Cauchy problem with the current as parameter and its mapping properties. The next section introduces a subdivision of the potential variables to get a computable version of the Cauchy problem. Section five is treating the singular cases of the general approximation by deriving stable, insensitive to rounding and higher order expressions for each case. The original Scharfetter-Gummel scheme is reproduced for the exponential function case. While the singular cases of the Cauchy problem result in simple current bounds in dependence of the arguments, the existence of a bounded logarithmic derivative allows to derive better bounds. These bounds are in some sense the closest point to analytic integration of the Cauchy problem reached up to now. Section six introduces the finite volume notation and states the discrete system of equations as numerical solutions of the Cauchy problem on each edge and as mean value theorem based expressions. The following section is establishing the existence of discrete bounded steady state solutions. The proofs are restricted to those parts which differ from the Boltzmann case. Section 
eight introduces the global Newton's method by using the chain rule and the unique solvability of the Cauchy problem on each edge. These relations are used in section nine to derive the properties of the global Jacobian. Especially negative and positive definite matrices result for the derivatives with respect to the electrostatic potential of the chemical potential related parts parts of the function, which correspond to elliptic operators with the proper sign in the analytic case. These results fill the gaps in section seven with respect to uniqueness of the discrete thermodynamic equilibrium. The diagonal contributions of the total charge and the reactions are easy to derive, have completely similar qualitative properties as in the Boltzmann case, hence they are not discussed in detail. Finally section ten is illustrating the goal to have the approximation errors of the functions $f(v)$ and $\mathcal{F}(v)$ and the related solution of the Cauchy problem as the only essential complexity changes compared with the Boltzmann case. Piecewise analytic integration avoids the necessary error control in case of numerically solving the integral equation for the edge current, which may be the winner on future computers. The considered approximations allow the treatment of large chemical potentials, hence strong degeneracy effects. Newton's method together with embedding and meshes defined by the accuracy requirements of the application problem (and not by stability related restrictions of the discretization) are prolongated from the Boltzmann to the Fermi-Dirac case, or more general to cases fulfilling the following basic assumptions for the chemical potential $v$ and the distribution functions $f, \mathcal{F}$ used in the paper:

$A_{1}:-\infty<v_{\min }<v<v_{\max }<\infty$,

$A_{2}: f(s)<f(t), s<t, 0<f^{\prime}(s) \leq f(s) \leq e^{s}$,

$A_{3}: \mathcal{F}(s)<\mathcal{F}(t), s<t, \quad 0<\mathcal{F}^{\prime}(s) \leq \mathcal{F}(s) \leq e^{s}$.

For instance the applications in mind

- very dense initial electron-hole plasma clouds and their faster decay in silicon detectors than predicted by the Boltzmann case [3] (deeper penetration of the electrostatic potential into the cloud in the Fermi case may be one part of the explanation),

- lasing hetero-structures using degenerate semiconductors are well in the range of the approximation of $\mathcal{F}_{1 / 2}$ given in section ten, and

- EDGM [4] like transport models in organic semiconductors

fulfill the assumptions.

\section{Short summary of the variational formulation}

The free energy functional $F$ is the sum of the convex chemical part $\Phi(p)$ and the interaction part $\Psi(p)$ :
$F(p)=\Phi(p)+\Psi(p)$

$p$ denotes the density of holes. $F(p)$ is minimized under particle conservation (Neumann boundary conditions)

$F^{\prime}(p)=\varphi=v+w$,

where $\varphi$ is the grand chemical (quasi-Fermi) potential,

$\Phi^{\prime}(p)=v, \quad \Psi^{\prime}(p)=w$

are the chemical and the interaction potential. $\Phi^{*}$ denotes the conjugate function

$p=\left(\Phi^{*}\right)^{\prime}(v)=\left(\Phi^{*}\right)^{\prime}(\varphi-w)=\left(\Phi^{\prime}\right)^{-1}(v)$,

$\Phi^{\prime^{-1}}$ is the inverse function in case that $\Phi^{\prime}(p)$ is monotone. Assuming

(a) conservation of mass in $\Omega \subset \mathbb{R}^{d}$,

(b) solutions along trajectories $t \mapsto p(t)$ satisfy the state equation with $\varphi(x)$,

(c) and the anti-gradient of $\varphi$ is the driving force

yields

$\frac{\partial p}{\partial t}+\nabla \cdot j_{p}=0$ in $\Omega, v \cdot j_{p}=0$ on $\partial \Omega$.

With (b), (c) and the chain rule (the constant diffusion coefficient, matrix is denoted by $D$ ), the particle current $j_{p}$ of the holes is

$-j_{p}=D\left(\Phi^{*}\right)^{\prime \prime}(\varphi-w) \nabla \varphi=D \frac{\partial p}{\partial v} \nabla \varphi$.

Finally,

$$
\begin{aligned}
-j_{p} & =D\left(\Phi^{*}\right)^{\prime \prime} \nabla(v+w), \\
& =D \nabla\left(\Phi^{*}\right)^{\prime}(v)+D\left(\Phi^{*}\right)^{\prime \prime}(v) \nabla w, \\
& =D \nabla p+D \frac{\partial p}{\partial v} \nabla w .
\end{aligned}
$$

Hence, in case of a variational formulation $A_{2}$ implies $A_{3}$ and the 'discrete chain rule' [5] is preserving the qualitative properties. In case the free energy functional is seen as a complete description, $D$ provides the time scale, else it may include density dependent corrections accounting for the missing parts in the free energy functional. For the general setting (not restricted to Fermi-Dirac integrals only) and a rigorous treatment including phase separation problems compare, e.g., [6-13] and cited literature. For relations with gradient structures, cf. [14].

When the equations are stated as a modification of the classical van Roosbroeck [1] equations (cf. [15]) the current expressions are 


$$
\begin{aligned}
-j_{\text {modclass }} & =\mu p \nabla \varphi_{p}, \\
-j_{\text {var }} & =D \frac{\partial p}{\partial v} \nabla \varphi_{p},
\end{aligned}
$$

and therefore

$$
\begin{aligned}
\frac{\mu p}{D \frac{\partial p}{\partial v}} & =1, \\
\frac{\mu}{D} & =\frac{\partial \ln p}{\partial v} .
\end{aligned}
$$

This is the generalized Einstein relation, which holds at thermodynamic equilibrium due to the total current equals zero. It is used for non-equilibrium states and relates the diffusion and drift current via the diffusion coefficient $D$ and the mobility $\mu$. Essentially it is a factor which can be attributed arbitrarily to drift and diffusion. The equations coincide if $\mu=D \frac{\partial p}{\partial v} / p$ is substituted.

If the densities are related to the chemical potential by exponential functions (Boltzmann statistics), the logarithmic derivative is equal to one and the Einstein relation is a constant, here one due to the scaling of the potentials.

The complete system reads in particle currents (electric charge densities, currents are multiplied by the charge per particle) and in more detail with respect to constants related to physics (density of states $N_{v}$ and $N_{c}$, band edges of valence and conduction band $E_{v}, E_{c}$ )

$p / N_{v}=\left(\Phi^{*}\right)^{\prime}\left(v_{p}\right)=\left(\Phi^{\prime}\right)^{-1}\left(v_{p}\right)=\mathcal{F}_{i}\left(v_{p}\right)$,

$v_{p}=\varphi_{p}-w+E_{v}$, while $\mathcal{F}_{i}(s)$ denotes the most relevant example, the Fermi-Dirac integral of index $i$. The index $i$ typically depends on the space dimension, e.g., $i=1 / 2$ for transport in $\mathbb{R}^{3}$. Accordingly,

$n / N_{c}=\left(\Phi^{*}\right)^{\prime}\left(v_{n}\right)=\left(\Phi^{\prime}\right)^{-1}\left(v_{n}\right)=\mathcal{F}_{i}\left(v_{n}\right)$,

$v_{n}=-\varphi_{n}+w-E_{c}, v_{p}$ and $v_{n}$ are the chemical potentials for holes and electrons of the material. Here $w$, and $\varphi_{p}$ and $\varphi_{n}$ denote the electrostatic and quasi-Fermi potentials, which are continuous functions. All potential-like quantities are 'measured' in units of the thermal voltage $U_{T}:=k_{B} T / q$, is the $k_{B}$ Boltzmann constant, $T$ is the temperature, and $q$ is the elementary charge. Thus

$$
\begin{aligned}
& -\nabla \cdot \varepsilon \nabla w=C-n+p, \\
& \quad \frac{\partial p}{\partial t}-\nabla \cdot D_{p}\left(\Phi^{*}\right)^{\prime \prime} \nabla \varphi_{p}=R(n, p)\left(1-r\left(\varphi_{p}-\varphi_{n}\right)\right), \\
& \quad \frac{\partial n}{\partial t}+\nabla \cdot D_{n}\left(\Phi^{*}\right)^{\prime \prime} \nabla \varphi_{n}=R(n, p)\left(1-r\left(\varphi_{p}-\varphi_{n}\right)\right)
\end{aligned}
$$

in the Lipschitzian domain $\Omega \subset \mathbb{R}^{d}$. For reasons of simplicity the boundary conditions are homogeneous Neumann for insulating parts and Dirichlet for contacts $\left(\Gamma_{D}=\cup_{i} \Gamma_{D_{i}}\right.$ with positive surface measure), where $w_{\text {applied }}$ is added to the contact potentials $\left(w_{i}=w_{i}^{*}+w_{\text {applied }_{i}}, \varphi_{p, i}=w_{\text {applied }_{i}}\right.$, $\left.\varphi_{n, i}=w_{\text {applied }_{i}}, x_{i} \in \Gamma_{D_{i}}\right)$. Hence, the densities at contacts are those of the thermodynamic equilibrium. This is defined by the nonlinear Poisson equation

$$
-\nabla \cdot \varepsilon \nabla w^{*}=C-n^{*}+p^{*}=C-\mathcal{F}\left(v_{n}^{*}\right)+\mathcal{F}\left(v_{p}^{*}\right)
$$

with $\varphi_{p}^{*}=\varphi_{n}^{*}=0$ and homogeneous Neumann boundary conditions. $w^{*}, v_{n}^{*}$ and $v_{p}^{*}$ denote the thermodynamic equilibrium solution. The recombination-generation expression $r(s)$ depends on the difference of the quasi-Fermi potentials. It is assumed: $r(s)$ is strictly monotone, $r(0)=1$, $\lim _{s \rightarrow-\infty} r(s)=0$, and $\lim _{s \rightarrow \infty} r(s)=\infty, R \geq 0$. This includes the classical Shockley-Read-Hall and Auger recombination-generation processes, but it is not anymore an expression of mass action law type. For the free energy expressions see, e.g., [7]. The discrete case fulfills analog discrete free energy relations in case it is applied to a variational formulation.

In the following the constants $N_{v}, N_{c}$ are set to one, $E_{v}$, $E_{c}$ to zero, re-substitution is not a problem. Not any qualitative property of the equations is changed. Furthermore $f_{i}\left(v_{i}\right):=D_{i}\left(\Phi^{*}\right)^{\prime \prime}\left(v_{i}\right), i=p, n$ describes the transport as long as assumption (2) holds-it is not necessary to suppose the variational formulation in that respect. The properties of the discretization are studied for $\varphi=v+w$ (holes) and the other case follows by proper substitutions, while $D$ or $\mu$ are included in $f$.

\section{Generalized Scharfetter-Gummel scheme}

The classical scheme discretizes all three parts of each continuity equation separately, providing local conservation of the total current (sum of displacement and charge density currents) as well as a dissipative approximations of the second order elliptic operator and the recombination-generation expressions. The second order operators of the classical equations can be integrated in closed form along an edge. Using the same assumption for the discrete electrostatic potential, $w_{e}=w_{l}+\frac{\delta w}{\delta x}\left(x-x_{l}\right)$ is a linear function along an edge $e$ connecting $x_{l}$ and $x_{r}$ with boundary values $w_{l}, w_{r}, v_{l}, v_{r}$, and $\varphi_{l}, \varphi_{r}$. The indices are skipped in the following by using $\delta x:=x_{r}-x_{l}, \delta \varphi:=\varphi_{r}-\varphi_{l}$ etc. The basic equation is the one-dimensional two-point boundary value problem

$\left.\frac{d}{d x}\left(f(v) \frac{d \varphi}{d x}\right)=0, x \in\right] x_{l}, x_{r}[$

with boundary values $\varphi_{l}, \varphi_{r}, f\left(v\left(x_{i}\right)\right)=f\left(v_{i}\right), i=l, r$. It is supposed that $f(v)$ is a rapidly varying coefficient function along an edge and density dependent parts of $D$ (or $\mu$ ) 
are included as long as assumption (2) holds. The subscripts $a, b$ are used for arbitrary (sub)intervals, while $l, r$ denote the integration interval of Eq. (12). The density is defined by $p=\mathcal{F}(v)$ and $f(v)$ can fulfill the relations imposed by the variational formulation or it may be modified in special models. Because the reaction terms depend on the difference of the quasi-Fermi potentials (up to positive pre-factors), the densities enter essentially via the right-hand side of the Poisson equation. Accordingly, the remaining part of the mobility is assumed to be slowly varying and its appropriate average is a constant pre-factor per edge in $f(v)$.

The first integration with an integration constant $j$, the edge current, yields

$f(v) \frac{d \varphi}{d x}=f(v) \frac{d(v+w)}{d x}=-j$,

hence

$f(v) \frac{d v}{d x} \approx-j-f(v) \frac{\delta w}{\delta x}$.

The approximation in (14) transforms (12) into a Cauchy problem with a special monotone dependence $f(v)$, which is neither changed by the constant $\delta w / \delta x$ nor by the constant $j$. Hence, for every Lipschitz continuous $f(v)$ and constant $j$ the Cauchy problem with the initial value $v_{l}$ has a unique solution $v(x, j)$. On the other hand, $j$ has to be chosen such that the boundary value $v\left(x_{r}\right)=v_{r}$ is fulfilled. Testing (13) with $\varphi^{\prime}$ results in the dissipation expression

$\int_{x_{l}}^{x_{r}} \varphi^{\prime 2} f(v) d x=-j\left(\varphi_{r}-\varphi_{l}\right)$.

The left hand side is nonnegative, hence $j$ has to change sign with $\delta \varphi$. The thermodynamic equilibrium is defined by $\delta \varphi=0$ for all edges in $\Omega$, hence $\varphi=$ const . Essentially one could allow such $w(x)$ that do not change the very implicit monotonicity of (13) and preserve the dissipativity of $j$ and the uniqueness of the thermodynamic equilibrium.

For special $f(v)$ and $w(x)$ one can get special cases of (13) in form of a Bernoulli or Riccatti equation, but separation of the variables in (14) and integration with respect to $d f$ was not successful up to now. Hence the aim is to give a construction for $j$ and (14) that results in a $j$ reproducing the dissipation of (14) and the unique thermodynamic equilibrium.

The second integration with respect to $v$ and $x$ yields an integral equation for $j$ and finite differences in the potentials:

$$
\begin{aligned}
& \int_{x_{l}}^{x_{r}} d x=\delta x \approx-\int_{v_{l}}^{v_{r}} \frac{d v}{\delta w / \delta x+j / f(v)}, \\
& \int_{v_{l}}^{v_{r}} \frac{f(v) d v}{j / J+f(v)}=-\delta w,
\end{aligned}
$$

or

$\delta v-\int_{v_{l}}^{v_{r}} \frac{j / J d v}{j / J+f(v)}=-\delta w$.

Hence, an implicit expression depending on $v_{l}, v_{r}, \delta w$, or $\delta \varphi$. While $J=\frac{\delta w}{\delta x}$ has the meaning of a drift current, $f_{0}=-\frac{j}{J}$ is the pole of the integrand. Due to $\frac{f(v)}{f(v)-f_{0}}=1+$ $\frac{f_{0}}{f(v)-f_{0}}$, the integrand is a monotone function for monotone $f(v)$ if $f_{0} \notin\left[f\left(v_{l}\right), f\left(v_{r}\right)\right]$. The special cases $\delta \varphi=0$ (hence $\delta w=-\delta v$, equilibrium due to (13)) and the vanishing drift current $\delta w=0$ define the limiting cases in the discussion of the mapping properties of Eq. (16). In the vanishing drift case a unique primitive of $f(v)$ defines $j$ uniquely, see (15).

Interchanging all boundary values changes only the sign of $j$, compare (5), (12), and (14), hence it is sufficient to consider the case $v_{l}<v_{r}$.

Theorem 1 Equation(16) determines the current $j$ uniquely if the value $f_{0}$ is chosen in dependence of $\delta w, v_{l}, v_{r}(\delta v>0$ fixed) by the following rules:

(i) $0<\delta w<\infty$ (meaning $0<\delta w / \delta v<\infty$, $\delta \varphi>0$ ): $f\left(v_{r}\right)<f_{0}<\infty$

(ii) $-\delta v<\delta w<0$ (meaning $-1<\delta w / \delta v<0, \delta \varphi>0$ ): $-\infty<f_{0}<0$,

(iii) $\delta w<-\delta v<0$ (meaning $-\infty<\delta w / \delta v<-1, \delta \varphi<$ $0): 0<f_{0}<f\left(v_{l}\right)$,

$j$ solves $I\left(f_{0}\right):=\int_{v_{l}}^{v_{r}} \frac{f(v) d v}{f(v)-f_{0}}=-\delta w$ and $\delta \varphi \cdot j \leq 0$.

Proof The rules and cases are cited in the following as (i), (ii), (iii). By the mean value theorem and Assumption $A_{2}$

$I\left(f_{0}\right)=\frac{\delta v f(\tilde{v})}{f(\tilde{v})-f_{0}}=-\delta w$

with exactly one $\tilde{v} \in] v_{l}, v_{r}$ [ for a fixed $f_{0}$. Further, it holds $\int_{a}^{b} f_{1}(s) d s>\int_{a}^{b} f_{2}(s) d s$ for $f_{1}>f_{2}$ on a subset of $[a, b]$ of positive measure.

(i) Due to the monotonicity of $f(v)$, the integrand is negative and strictly monotonously decreasing for $f(v) \in$ ]0, $f_{0}\left[\right.$ for all $f_{0}>f\left(v_{r}\right)$. For $\left.f_{0} \in\right] f\left(v_{r}\right), \infty[, I$ is a monotonous mapping of $] f\left(v_{r}\right), \infty[$ onto $]-\infty, 0[$, hence there is exactly one $f_{0}^{*}$ with $I\left(f_{0}^{*}\right)=-\delta w$.

(ii) Now the integrand is a positive, strictly increasing function for $f(v)>0$, hence (18) holds and, with respect to $f_{0}$, it is a monotonous mapping of $]-\infty, 0[$ onto $] 0, \delta v[$. Thus, (18) is fulfilled for exactly one $\left.f_{0}^{*} \in\right]-\infty, 0[$.

(iii) The integrand is now a positive, strictly decreasing function and the arguments apply again for the monotonous mapping $\left.f_{0} \in\right] 0, f\left(v_{l}\right)$ [ onto $] \delta v, \infty[$. 
$j=0$ implies $\delta v=-\delta w$ in (16), hence $\delta \varphi=0$ according to (5). Due to the uniqueness for $\delta v \neq-\delta w$ and $j \neq 0$, the equilibrium is uniquely defined by $\delta \varphi=0$.

For $\delta w \rightarrow \infty$ holds $f_{0} \rightarrow f\left(v_{r}\right)$ and $\delta w \rightarrow-\infty$ gives $f_{0} \rightarrow f\left(v_{l}\right)$. Hence, $h$ with $h(\delta w):=-f_{0} J=j$ is a monotone mapping from $]-\infty, \infty[$ onto $]-\infty, \infty[$ and it is bijective. To show $\delta \varphi \cdot j \leq 0$ one calculates the sign of $\delta \varphi f_{0}^{*}$ in each of the cases:

(i) $f_{0}^{*}>0, \delta w>0$, hence $j<0$ and $\delta \varphi>0$,

(ii) $f_{0}^{*}<0, \delta w<0$, hence $j<0$ and $\delta \varphi>0$,

(iii) $f_{0}^{*}>0, \delta w<0$, hence $j>0$ and $\delta \varphi<0$.

Remark 1 Choosing $\left.f_{0} \in\right] f\left(v_{l}\right), f\left(v_{r}\right)[$ and $\delta w \in]-\infty, \infty[$ gives $\delta \varphi \in]-\infty, \infty\left[\right.$ and $f_{0}>0$, so that $\delta \varphi \cdot j \leq 0$ can not be true for all arguments.

\section{Introduction of a subdivision on $\left[v_{l}, v_{r}\right]$ and approximation on the subintervals}

A subdivision of $v_{l}=v_{0}<v_{1}<\cdots<v_{i}<\cdots<v_{k+1}=$ $v_{r}, \delta v_{i}=v_{i+1}-v_{i}=\beta_{i} \delta v$ is introduced to get a computable approximation of the integral. $\beta_{i}$ define the partitioning of the straight line from $v_{l}$ to $v_{r}$ in the $\varphi w$-plane, hence the projections satisfy $\delta \varphi_{i}=\beta_{i} \delta \varphi$ and $\delta w_{i}=\beta_{i} \delta w$. Because $f_{0} \notin\left[f\left(v_{l}\right), f\left(v_{r}\right)\right]$ and

$\sum_{i=0}^{k} \int_{v_{i}}^{v_{i+1}} \frac{f(v) d v}{f(v)-f_{0}}=-\delta w$,

there exists for each interval $\left[v_{i}, v_{i+1}\right]$ one $\tilde{v}_{i} \in\left[v_{i}, v_{i+1}\right]$ with $f_{i}:=f\left(\tilde{v}_{i}\right)$ such that

$\int_{v_{i}}^{v_{i+1}} \frac{f(v) d v}{f(v)-f_{0}}=\frac{f_{i} \delta v \beta_{i}}{f_{i}-f_{0}}=\frac{f_{i} \delta v_{i}}{f_{i}-f_{0}}$

holds by the mean value theorem.

The only change to the previous section is the introduction of the decomposition of unity by $\beta_{i}$, given by the intervals $\delta v_{i}$, and $\zeta_{i}>0$ with $\sum_{i=0}^{k} \zeta_{i}=1$. Hence, $f_{0}^{*}$ can be inserted that solves

$\sum_{i=0}^{k} \frac{f_{i} \beta_{i}}{f_{i}-f_{0}^{*}}=-\frac{\delta w}{\delta v} \sum_{i=0}^{k} \zeta_{i}, \frac{f_{i} \beta_{i}}{f_{i}-f_{0}^{*}}=-\frac{\delta w}{\delta v} \zeta_{i}$,

because it is just a reformulation of $I\left(f_{0}^{*}\right) / \delta v . \zeta_{i}$ is the relative contribution of the partial integral $i$ to the sum for a given $f_{0} \cdot \sum_{i=0}^{k} \zeta_{i}=1$ holds for $f_{0}=f_{0}^{*}$. The next step is to replace $f(v)$ by piecewise differentiable, monotone approximations $f_{i}(v)$, $\sum_{i=0}^{k} \int_{v_{i}}^{v_{i+1}} \frac{f_{i}(v) d v}{f_{i}(v)-f_{0}}=-\delta w$,

$f_{0}$ identical for all $i$. The $f_{i}$ are fulfilling the basic assumptions $A_{1}, A_{2}$ (on each subinterval) and coincide with $f\left(v_{i}\right)$ at the collocation points $v_{i}$ :

$$
\begin{aligned}
A_{4}: f_{0}\left(v_{0}\right) & =f\left(v_{l}\right), \ldots, f_{i}\left(v_{i+1}\right)=f_{i+1}\left(v_{i+1}\right) \\
& =f\left(v_{i+1}\right), \ldots, f_{k}\left(v_{k+1}\right)=f\left(v_{r}\right) .
\end{aligned}
$$

Due to the construction, Theorem 1 holds for this approximation of $f(v)$. On each interval one can chose an approximation allowing integration in closed form.

\section{Special cases}

The main purpose of this section is to derive expressions for special cases that allow a sufficient overlap of the standard approximation of the integral equation. The reason is twofold: the influence of rounding errors is one aspect, the second one is sufficiently smooth function definitions that do not deteriorate the convergence of the global Newton method. Before special cases, like $v_{r} \approx v_{l}$, are treated, the verification of the discretization scheme in case of the exponential function is considered:

\subsection{Boltzmann statistics}

$$
\begin{aligned}
-\delta \varphi= & f_{0} \int_{v_{l}}^{v_{r}} \frac{d v}{f(v)-f_{0}}=f_{0} \int_{v_{l}}^{v_{r}} \frac{d v}{e^{v}-f_{0}} \\
= & \left.\left(\ln \left(e^{v}-f_{0}\right)-v\right)\right|_{v_{l}} ^{v_{r}}=\ln \frac{e^{v_{r}}-f_{0}}{e^{v_{l}}-f_{0}}-\delta v, \\
& \left(e^{v_{l}}-f_{0}\right) e^{-\delta w}=e^{v_{r}}-f_{0}, \\
f_{0}= & \frac{e^{v_{r}}-e^{v_{l}-\delta w}}{1-e^{-\delta w}}=-\frac{e^{v_{r}}}{e^{-\delta w}-1}-\frac{e^{v_{l}}}{e^{\delta w}-1} \\
= & \frac{e^{v_{r}}\left(1-e^{\delta w}\right)+e^{v_{l}}\left(1-e^{-\delta w}\right)}{2(1-\cosh (\delta w)}=-\frac{j \delta x}{\delta w},
\end{aligned}
$$

where nominator and denominator vanish for $\delta w=0$. For the current

$j \delta x=e^{v_{l}} \mathrm{~b}(\delta w)-e^{v_{r}} \mathrm{~b}(-\delta w)$

one gets back the Bernoulli functions and a smooth dependence of the parameters. The current $j$ vanishes for

$$
\begin{aligned}
& e^{\delta w} \frac{\mathrm{b}(\delta w)}{\mathrm{b}(-\delta w)}=e^{\delta \varphi}, \\
& \quad \frac{\delta w\left(e^{-\delta w}-1\right)}{e^{-\delta w}\left(e^{\delta w}-1\right)(-\delta w)}=1=e^{\delta \varphi},
\end{aligned}
$$

hence at the thermodynamic equilibrium. 


\subsection{Bounded logarithmic derivative}

Here it is supposed that $f(v)$ has a logarithmic derivative fulfilling

$0<\varepsilon<f^{\prime}(v) / f(v) \leq 1$ for $v_{\min }<v<v_{\max }$.

This is true for Fermi-Dirac integrals of index $i, i$ real, $i \geq$ -1 , because $f(s)=\mathcal{F}_{-1}(s) \approx 1-e^{-s}$ for large arguments.

Now the integral can be rewritten as

$$
\begin{aligned}
\int_{v_{l}}^{v_{r}} \frac{d v}{f(v)-f_{0}} & =\int_{v_{l}}^{v_{r}} \frac{f f^{\prime} d v}{f f^{\prime}\left(f(v)-f_{0}\right)} \\
& =\int_{f_{l}}^{f_{r}} \frac{f d f}{f f^{\prime}\left(f(v)-f_{0}\right)} \\
& =\int_{f_{l}}^{f_{r}} \frac{d f}{(\ln f)^{\prime} f(v)\left(f(v)-f_{0}\right)}:=K .
\end{aligned}
$$

Due to the generalized mean value theorem

$\int_{a}^{b} h(x) g(x) d x=h(\xi) \int_{a}^{b} g(x) d x$,

there exists at least one $\xi \in[a, b]$ if $h(x)$ is continuous and $g(x)$ does not change sign in $[a, b]$. Hence, $K$ can be bounded by

$$
\begin{aligned}
L & \leq K<\frac{1}{\varepsilon} L, \\
L & :=\int_{f_{l}}^{f_{r}} \frac{d f}{f(v)\left(f(v)-f_{0}\right)}, \\
& =\frac{1}{f_{0}} \ln \frac{f_{l}\left(f_{r}-f_{0}\right)}{f_{r}\left(f_{l}-f_{0}\right)}, \quad 0<f_{l}<f_{r}
\end{aligned}
$$

and $\left(f_{r}-f_{0}\right) /\left(f_{l}-f_{0}\right)>0$ because $f_{0} \notin\left[f_{l}, f_{r}\right]$. Denoting the mean value of one over the logarithmic derivative in Eq. (24) by $\left\langle\frac{1}{(\ln f(v))^{\prime}}\right\rangle$ and due to $-\delta \varphi=f_{0} K$ yields

$$
\begin{aligned}
K & =\left\langle\frac{1}{(\ln f(v))^{\prime}}\right\rangle L, \\
-\delta \varphi & =\left\langle\frac{1}{(\ln f(v))^{\prime}}\right\rangle \ln \frac{f_{l}\left(f_{r}-f_{0}\right)}{f_{r}\left(f_{l}-f_{0}\right)} .
\end{aligned}
$$

Both sides vanish at equilibrium and $\left\langle\frac{1}{(\ln f(v))^{\prime}}\right\rangle>0$. Replacing the mean value by $1 / c$ results in

$$
\begin{aligned}
e^{-\delta \varphi c} & =\frac{f_{l}\left(f_{r}-f_{0}\right)}{f_{r}\left(f_{l}-f_{0}\right)}, \\
e^{-\delta \varphi c} f_{r}\left(f_{l}-f_{0}\right) & =f_{l}\left(f_{r}-f_{0}\right), \\
f_{0} & =\frac{\left(e^{-\delta \varphi c}-1\right) f_{r}}{\left(e^{-\delta \varphi c} \frac{f_{r}}{f_{l}}-1\right)} .
\end{aligned}
$$

The nominator vanishes at the thermodynamic equilibrium, while $f_{0}$ moves to infinity for vanishing denominator, and $f_{0}=f_{r}=f_{l}$ in case of $f_{l}=f_{r}(\delta v=0)$ and $e^{-\delta \varphi c}=1$. The qualitative properties are similar to that of the Boltzmann case. Inserting the known extreme values of the $f(v) / f^{\prime}(v)$, $v \in\left[v_{l}, v_{b}\right]$ into Eq. (25) improves the bounds for $f_{0}$, and, hence, $j$.

\subsection{The case $\delta w \approx 0$}

The case of a finite $j$ and $\delta w \approx 0$ (small drift contribution) simplifies the original equation: $-j \approx f(v) d v / d x$, hence $-j \delta x=F\left(v_{r}\right)-F\left(v_{l}\right), F(v)=\int f(v) d v$ in case $\delta w=0$. This case is consistent with an expansion of the denominator assuming $\left|f_{0}\right|>>f\left(v_{r}\right)$, hence substitution of

$\frac{1}{f(v)-f_{0}}=\frac{-1}{f_{0}} \frac{1}{1-\frac{f(v)}{f_{0}}}=\frac{-1}{f_{0}} \sum_{i=0}^{\infty}\left(\frac{f(v)}{f_{0}}\right)^{i}$

yields

$\delta w f_{0}=-j \delta x=\int_{v_{l}}^{v_{r}} f(v) \sum_{i=0}^{\infty}\left(\frac{f(v)}{f_{0}}\right)^{i} d v$

if $|\delta w|<<\min (|\delta v|,|\delta \varphi|)$ and $|\delta v| \approx|\delta \varphi|$. An additionally small $|\delta v|$ means a situation close to equilibrium and is considered below.

\subsection{The case $\delta v \approx 0$}

In case $\delta v \approx 0$ one can assume that all extrema of the densities are represented approximately by the grid. The case $\delta v \approx 0$ is just a reduction to the constant coefficient case and the one-dimensional equation $\left(f(v) \varphi^{\prime}\right)^{\prime}=0$ results in $-j \delta x=\tilde{f}(v) \delta \varphi$, where $\tilde{f}(v)$ is a suitable mean value of $f(v)$. In many situations one can assume that deviations from Boltzmann statistics are small for some $v_{B}$ and $v_{r}<v_{B}$. (This case is handled in Sect. 10.1). Hence, $\delta v \approx 0$ implies $f_{r}-f_{l}<<f_{l}$ and the interesting cases are

$f_{0}=f_{l}-\varepsilon, \quad f_{0}=f_{r}+\varepsilon$,

$\delta f_{l}=f(v)-f_{l}>0, \quad \delta f_{r}=f(v)-f_{r}<0$

with $v \in\left[v_{l}, v_{r}\right]$ and $\left|\delta f_{i} / f_{i}\right|<<1, i=l, r$. The equality

$\int_{v_{l}}^{v_{r}} \frac{f_{0} d v}{f-f_{0}}=-\delta \varphi$

is equivalent to

$$
\begin{aligned}
\int_{v_{l}}^{v_{r}} \frac{d v}{f / f_{0}-1} & =\int_{v_{l}}^{v_{r}} \frac{d v}{\left(1+\delta f_{r} / f_{r}\right) /\left(1+\varepsilon / f_{r}\right)-1} \\
& =\left(f_{r}+\varepsilon\right) \int_{v_{l}}^{v_{r}} \frac{d v}{\delta f-\varepsilon} .
\end{aligned}
$$


The mean value theorem gives

$$
\frac{f_{r} \frac{\delta v}{\delta \varphi}+\delta f_{r}\left(v_{l}\right)}{1-\frac{\delta v}{\delta \varphi}}<\varepsilon<\frac{f_{r} \frac{\delta v}{\delta \varphi}}{1-\frac{\delta v}{\delta \varphi}} .
$$

These bounds and Eq. (28) result in a case handled in Sect. 10.3 , a symmetric expansion of the integral with respect to the difference of the bounds should be used. The situation $f_{0}=f_{l}-\varepsilon<0$ can be handled similarly.

The other option is to evaluate numerical integration formulas analytically and to bootstrap via different formulas. The starting point is the simplest version of the trapezoidal rule,

$f_{0}\left(\frac{1}{f_{l}-f_{0}}+\frac{1}{f_{r}-f_{0}}\right) \frac{\delta v}{2} \approx-\delta \varphi$,

$f_{0}^{2}(\delta \varphi-\delta v)+f_{0} \frac{f_{l}+f_{r}}{2}(\delta v-2 \delta \varphi)+\delta \varphi f_{l} f_{r}=0$.

The first order approximation $\left(\bar{f}=\left(f_{l}+f_{r}\right) / 2, \delta f=\right.$ $\left.\left(f_{r}-f_{l}\right)\right)$ is

$f_{0,1}=\frac{\delta \varphi\left(\bar{f}^{2}-(\delta f / 2)^{2}\right)}{\bar{f}(2 \delta \varphi-\delta v)}$

and the second order approximation reads

$$
\begin{aligned}
f_{0,2 \pm}= & (-2 \delta \varphi \bar{f}+\bar{f} \delta v \pm \\
& \left.\sqrt{\delta f^{2} \delta \varphi(\delta \varphi-\delta v)+\bar{f}^{2} \delta v^{2}}\right) /(2(\delta \varphi-\delta v)) .
\end{aligned}
$$

Here the sign is defined by the cases $(i, i i, i i i)$. During computations the correctness of both formulas with respect to $f_{l}, f_{r}$ can be easily checked. If at least (32) is correct, the third order equation resulting from Eq. (28) using the trapezoidal rule with two (or more) intervals can be solved by Newton's method without any transformation of the formula using $f_{0,2 \pm}$ as initial guess.

The numerical integration is of general interest. The 'expansion-like' expressions show different forms of the deviation of the pole from the mean value $\bar{f}$, hence error control is an issue for expansions and numerical integration.

\subsection{The equilibrium}

The equilibrium and the computation of the related derivatives is based on the expansion of the integral equation for sufficiently small, scaled currents $f_{0}$. It yields

$$
\int_{v_{l}}^{v_{r}} \sum_{i=0}^{\infty}\left(\frac{f_{0}}{f(v)}\right)^{i} d v=-\delta w
$$

hence using monotonicity and $0 \leq\left|f_{0}\right|<f\left(v_{l}\right)<f\left(v_{r}\right)$ results in a convergent power series of the integrand and the zero-order term is reproducing the equilibrium condition. The original integral equation can result in large rounding errors for the close to equilibrium cases. Subtracting the equilibrium condition or the zero order term in (33) yields

$\int_{v_{l}}^{v_{r}} \sum_{i=1}^{\infty}\left(\frac{f_{0}}{f(v)}\right)^{i} d v=\sum_{i=1}^{\infty} f_{0}^{i} \int_{v_{l}}^{v_{r}} \frac{d v}{f^{i}(v)}=-\delta \varphi$.

This is the defining nonlinear equation for $f_{0}$, which is truncated accordingly to the precision needed. Equation (34) can be evaluated easily: the integrals are independent of $f_{0}$ and can be precomputed for all approximation intervals (including the sums, if the data is ordered with respect to the start intervals $v_{l}$, which is an advantage with respect to data locality anyway). Hence at most start and end interval have to be evaluated and summed. Very close to equilibrium it is sufficient to keep the first order term, hence

$j \approx \frac{\delta w}{\delta x} \frac{1}{\int_{v_{l}}^{v_{r}} \frac{d v}{f(v)}} \delta \varphi$.

Due to $\delta \varphi \approx 0$ it holds $-\delta w=\delta v-\delta \varphi$ with $\delta v>$ 0 . Hence using the mean value of the denominator integral $\bar{f} \delta v=\int_{v_{l}}^{v_{r}} \frac{d v}{f(v)}$

$j \approx-\frac{\delta \varphi}{\bar{f} \delta x}$.

The derivative with respect to $\delta w$ vanishes due to the remaining factor $\delta \varphi=0$.

The chosen approximations can be evaluated up to four terms without much effort. Because $f_{0}<c f\left(v_{l}\right)$ for sufficiently small $c(c<1 / 1000)$, monotonicity of the expansion is guaranteed for $v \in\left[v_{l}, v_{r}\right]$ and the remaining term is of order $10^{-15}$. No problems in solving that equation by two steps of an undamped Newton's method are expected if the solution of the truncated linear equation is used as an initial guess. For $\delta v \approx 0$ an expansion at $\bar{v}$ simplifies the problem further, see above.

\section{Discretization and finite volume notation}

The basic assumptions of the finite volume discretization are:

- Gauss' theorem holds, hence one is able to discretize solutions with bounded variation and is not forced to resolve internal and boundary layers with very small feature size due to stability reasons-other reasons may or may not exist; they can depend on solution methods and the application for instance,

- boundary conforming Delaunay meshes $[16,17]$ cover the bounded polyhedral domain $\Omega=\cup_{l} \mathbf{E}_{l}^{N}$, 
where $\mathbf{E}_{l}^{N}$ denotes $l$ th $N$-dimensional simplex. The notation follows [12] and aims the similarity with the analytic one, hence $G$ stands for 'Gradient' $\nabla, G^{T}$ for $\nabla \cdot$, and $-\nabla \cdot \varepsilon \nabla$ 'translates' into $G^{T}[\varepsilon] G$.

$G$ includes the geometric weights $[\gamma]$ and contributions of boundary conditions while $\tilde{G}$ is a difference matrix. $\tilde{G}$ maps a vector $\mathbf{u}$ defined on the nodes of $\mathbf{E}_{l}^{N}$ as edge differences onto the edges of $\mathbf{E}_{l}^{N}$, and $\tilde{G}^{T}$ maps the differences with different signs back to the nodes of $\mathbf{E}_{l}^{N}$. The advantage is an explicit product of first order differences and 'integration by parts' is as simple as in the analytic case. The summation order does not matter and summing over all simplices or only one edge is not indicated but is clear from the context.

The integration over the intersection of each simplex and the Voronoi volumes associated with its nodes results in the weight $[V], V_{i}>0$, while density contributions are summed per Voronoi volume intersection and simplex, because in case of hetero structures (not dealt with in detail here) an explicit dependence of the material of each simplex has to be taken into account and can not be factored into a global diagonal matrix as in the Boltzmann case. Using [] to denote diagonal matrices gives

$G^{T}[\varepsilon] G \mathbf{w}=[V](\mathbf{C}-\mathbf{n}+\mathbf{p})$.

The nonlinear Poisson equation remains unchanged up to the definition of $n$ and $p$. The continuity equations have to be expressed by the implicitly defined edge currents. Equation (16), the cases $(i, i i, i i i)$, and the approximation used due to the boundary values result in

$\tilde{G}^{T}[\gamma] \mathbf{j}\left(\mathbf{v}_{\mathbf{p}}, \tilde{G} \varphi_{\mathbf{p}}\right)=[V][R([n],[p])]\left(\mathbf{1}-\mathbf{r}\left(\left[\varphi_{\mathbf{p}}-\varphi_{\mathbf{n}}\right]\right)\right)$,

$-\tilde{G}^{T}[\gamma] \mathbf{j}\left(\mathbf{v}_{\mathbf{n}}, \tilde{G} \varphi_{\mathbf{n}}\right)=[V][R([n],[p])]\left(\mathbf{1}-\mathbf{r}\left(\left[\varphi_{\mathbf{p}}-\varphi_{\mathbf{n}}\right]\right)\right)$.

Because the mean value theorem based currents include a factor $1 / \delta x$ the weights that are used in explicit form only in the continuity equations differ from those introduced in [12] by a factor $\delta x$. Using the standard expression for the current resulting from

$$
\begin{aligned}
& \int_{v_{l}}^{v_{r}} \frac{f(v) d v}{f(v)-f_{0}}=-\delta w \\
& \quad f_{0} \delta v=-\left(f(\tilde{v})-f_{0}\right) \delta w
\end{aligned}
$$

hence

$$
-j=\frac{f(\tilde{v})}{\delta x} \delta \varphi
$$

and remembering the negative definite Eq. (12) for the edge problem yields the final form of Eq. (37) for the proofs
$\tilde{G}^{T}[\gamma]\left[\frac{f\left(\tilde{v}_{p}\right)}{\delta x}\right] \tilde{G} \varphi_{\mathbf{p}}=[V][R([n],[p])]\left(\mathbf{1}-\mathbf{r}\left(\varphi_{\mathbf{p}}-\varphi_{\mathbf{n}}\right)\right)$,

where $\left[f\left(\tilde{v}_{p}\right)\right]$ denotes the intermediate value per edge solving Eq. (39). Assuming a boundary conforming Delaunay grid and $f\left(\tilde{v}_{p}\right)>0$ guarantee a weakly diagonally dominant S-matrix. The strict monotonicity and the mean value estimates are sufficient to prove existence of steady state solutions for that system.

Remark 2 Due to the assumptions, $j=0$ iff $\delta \varphi=0$, hence $\varphi=$ const.

\section{Existence of bounded steady state solutions}

Let

$$
\begin{aligned}
& V_{i} g_{i}\left(w_{i}, \varphi_{p, i}, \varphi_{n, i}\right):=\{[V](\mathbf{C}-\mathbf{n}+\mathbf{p})\}_{i}= \\
& \quad V_{i}\left(C_{i}-\mathcal{F}\left(-\varphi_{n, i}+w_{i}\right)+\mathcal{F}\left(\varphi_{p, i}-w_{i}\right)\right), \quad i \in[1, M],
\end{aligned}
$$

be the $i$ th component of the right-hand side of Eq. (36) and $M$ the number of nodes in the discretization. Because of $A_{3} w_{i} \mapsto g_{i}\left(w_{i}, \varphi_{p_{i}}, \varphi_{n_{i}}\right)$ is a strictly monotone mapping from $\mathbb{R}$ onto $\mathbb{R} \forall i$. Hence, $g_{i}\left(\cdot, \varphi_{p_{i}}, \varphi_{n_{i}}\right)=0$ has a unique solution $w_{i}$ for given $\varphi_{p, i}, \varphi_{n, i}$. To construct upper and lower bounds for $w$ the monotone increasing function $h(s):=\mathcal{F}(s)-\mathcal{F}(-s)$ is used. Let $\check{C}=\min (\mathbf{C})$, $\hat{C}=\max (\mathbf{C})$ denote the minimum and maximum of the doping $\forall i$, hence $\check{w}^{*} \leq h^{-1}(\check{C}) \leq w^{*} \leq h^{-1}(\hat{C}) \leq \hat{w}^{*}$ are the minimum, maximum of the built-in potential.

Assuming that the quasi-Fermi potentials are bounded by the Dirichlet data

$\underline{w}=\min \left(0, w_{\text {applied }}\right), \quad \bar{w}=\max \left(0, w_{\text {applied }}\right)$,

which are the bounds for the applied voltages, one has

$\check{\varphi}_{p}=\underline{w}, \quad \hat{\varphi}_{p}=\bar{w}, \quad \check{\varphi}_{n}=\underline{w}, \quad \hat{\varphi}_{n}=\bar{w}$,

hence

$\check{\varphi}_{p} \leq \varphi_{p} \leq \hat{\varphi}_{p}, \quad \check{\varphi}_{n} \leq \varphi_{n} \leq \hat{\varphi}_{n}$

Now bounds for $w$ can be constructed using the right hand side of the Poisson equation for nonequilibrium states: let $i$ be the vertex where $\hat{C}$ is realized, hence the right hand side of

$\mathcal{F}\left(-\varphi_{n, i}+w_{i}\right)=\hat{C}+\mathcal{F}\left(\varphi_{p, i}-w_{i}\right)$

is maximized for

$\mathcal{F}(s) \leq \hat{C}+\mathcal{F}\left(\hat{\varphi}_{p}-\underline{w}-\check{w}^{*}\right)$, 
$s=\mathcal{F}^{-1}\left(\hat{C}+\mathcal{F}\left(\hat{\varphi}_{p}-\underline{w}-\check{w}^{*}\right)\right)$,

$\hat{w} \leq \mathcal{F}^{-1}\left(\hat{C}+\mathcal{F}\left(\hat{\varphi}_{p}-\underline{w}-\check{w}^{*}\right)\right)+\hat{\varphi}_{n}$

$=\mathcal{F}^{-1}\left(\hat{C}+\mathcal{F}\left(\bar{w}-\underline{w}-\check{w}^{*}\right)\right)+\bar{w}$.

The lower bound $\check{w}$ is derived similarly,

$\check{w} \geq-\mathcal{F}^{-1}\left(|\check{C}|+\mathcal{F}\left(-\underline{w}+\hat{w}+\hat{w}^{*}\right)\right)+\underline{w}$.

Theorem 2 Let $\varphi_{\mathbf{p}}, \varphi_{\mathbf{n}}$ be given vectors with $\check{w} \leq \varphi_{p, i} \leq \hat{w}$, $\check{w} \leq \varphi_{n, i} \leq \hat{w}$. Then $\mathbf{w}$ is the unique solution of Eq. (36) and fulfills the bounds $\breve{w} \leq w_{i} \leq \hat{w}$.

Proof Existence: $G^{T}[\varepsilon] G$ is a weakly diagonally dominant M-matrix and $\partial\left(\mathcal{F}\left(-\varphi_{n, j}+w_{j}\right)-\mathcal{F}\left(\varphi_{p, j}-w_{j}\right)\right) / \partial w_{j}:=$ $g_{w, j}>0$ holds $\forall j \in[1, M]$, hence a unique positive inverse of $G^{T}[\varepsilon] G+[V]\left[g_{w}\right]$ exists for any $\mathbf{w}$ fulfilling the bounds. Hence, a damped Newton's method can be used to construct a unique $\mathbf{w}$ fulfilling the equation and the boundary conditions.

Bounds: assume $w_{i}>\hat{w}$ for some $i, x_{i} \notin \Gamma_{D}$, hence testing with the positive part $(\mathbf{w}-\hat{w})^{+T}$, or the weak discrete maximum principle, results in

$$
\begin{aligned}
& (\mathbf{w}-\hat{w})^{+T} G^{T}[\varepsilon] G \mathbf{w}= \\
& \quad(\mathbf{w}-\hat{w})^{+T}[V]\left(\mathbf{C}-\mathcal{F}\left(-\varphi_{n, i}+w_{i}\right)+\mathcal{F}\left(\varphi_{p, i}-w_{i}\right)\right) .
\end{aligned}
$$

$(\mathbf{w}-\hat{w})^{+T} G^{T}[\varepsilon] G \mathbf{w}>0$ but $\mathbf{C}-\mathcal{F}\left(-\varphi_{n, i}+w_{i}\right)+\mathcal{F}\left(\varphi_{p, i}-\right.$ $\left.w_{i}\right)<0 \forall i$ with $w_{i}>\hat{w}$, hence a contradiction. The lower bound follows by testing with $(\mathbf{w}-\check{w})^{-T}$.

These bounds for $\mathbf{w}$ provide bounds on $\varphi_{\mathbf{p}}, \varphi_{\mathbf{n}}$, Eq. (45). The next step is to verify these bounds.

Theorem 3 Let $\mathbf{w}\left(\varphi_{\mathbf{p}}^{\mathbf{0}}, \varphi_{\mathbf{n}}^{\mathbf{0}}\right), \varphi_{\mathbf{p}}^{\mathbf{0}}$, $\varphi_{\mathbf{n}}^{\mathbf{0}}$ be solutions of Eqs. (37) and (51), the analog equation for $\varphi_{\mathbf{n}}$ with frozen coefficients and $\varphi_{\mathbf{p}}$ the solution of

$$
\begin{aligned}
& \tilde{G}^{T}[\gamma]\left[\frac{f\left(\tilde{v}_{p}\right)}{\delta x}\right] \tilde{G} \varphi_{\mathbf{p}} \\
& =[V]\left[R\left(\left[n^{0}\right],\left[p^{0}\right]\right)\right]\left(\mathbf{1}-\mathbf{r}\left(\varphi_{\mathbf{p}}-\varphi_{\mathbf{n}}^{\mathbf{0}}\right)\right),
\end{aligned}
$$

$\mathbf{v}_{\mathbf{p}}^{\mathbf{0}}=\varphi_{\mathbf{p}}^{\mathbf{0}}-\mathbf{w}, \mathbf{p}^{\mathbf{0}}=\mathbf{p}\left(\mathbf{v}_{\mathbf{p}}^{\mathbf{0}}\right), \mathbf{v}_{\mathbf{n}}^{\mathbf{0}}=\mathbf{w}-\varphi_{\mathbf{n}}^{\mathbf{0}}, \mathbf{n}^{\mathbf{0}}=\mathbf{n}\left(\mathbf{v}_{\mathbf{n}}^{\mathbf{0}}\right)$, but $f\left(\tilde{v}_{p_{i}}\right)$ is the solution of Eq. (39) for all edges $i$ with frozen coefficients. The initial guesses $\varphi_{\mathbf{p}}^{\mathbf{0}}, \varphi_{\mathbf{n}}^{\mathbf{0}}$ are constructed such that they are bounded by the Dirichlet values. The solution $\varphi_{\mathbf{p}}$ fulfills the bounds (45).

Proof Assume $\varphi_{p_{i}}>\bar{w}$ for some interior vertices. Testing with $\left(\varphi_{\mathbf{p}}-\bar{w}\right)^{+T}$ results in

$$
\left(\varphi_{\mathbf{p}}-\bar{w}\right)^{+T} \tilde{G}^{T}[\gamma]\left[\frac{f\left(\tilde{v}_{p}\right)}{\delta x}\right] \tilde{G} \varphi_{\mathbf{p}}>0,
$$

zero is excluded by the construction of $\tilde{v}_{p}$ and $A_{2}$, and

$\left(\varphi_{\mathbf{p}}-\bar{w}\right)^{+T}[V]\left[R\left(\left[n^{0}\right],\left[p^{0}\right]\right)\right]\left(\mathbf{1}-\mathbf{r}\left(\varphi_{\mathbf{p}}-\varphi_{\mathbf{n}}^{\mathbf{0}}\right)\right)<0$

because $\left(\varphi_{p_{i}}-\varphi_{n_{i}}^{0}\right)>0$ for $\left(\varphi_{\mathbf{p}}-\bar{w}\right)^{+T}>0$, hence $r\left(\varphi_{p_{i}}-\right.$ $\left.\varphi_{n_{i}}^{0}\right)>1$, a contradiction.

Assuming now $\left(\varphi_{\mathbf{p}}-\underline{w}\right)^{-T}<0$ for some interior vertices does not change the sign of the left-hand side, but $\left(\varphi_{p_{i}}\right.$ $\left.\varphi_{p_{i}}^{0}\right)<0$ for all vertices where $\left(\varphi_{\mathbf{p}}-\underline{w}\right)^{-T}<0$, hence $r\left(\varphi_{p_{i}}-\varphi_{n_{i}}^{0}\right)<1$, hence

$\left(\varphi_{\mathbf{p}}-\underline{w}\right)^{-T}[V]\left[R\left(\left[n^{0}\right],\left[p^{0}\right]\right)\right]\left(\mathbf{1}-\mathbf{r}\left(\varphi_{\mathbf{p}}-\varphi_{\mathbf{n}}^{\mathbf{0}}\right)\right)<0$

yields a contradiction again. Solutions $\varphi_{\mathbf{p}}$ have to fulfill the bounds. The same holds for $\varphi_{\mathbf{n}}$ due to the sign changes of $\varphi_{\mathbf{n}}$, in the recombination term, and in the equation.

Theorem 4 On any connected, boundary conforming Delaunay mesh with $M$ vertices the problem (36), (37), and (38) with positive Dirichlet boundary measure has at least one solution. It fulfills the bounds (43), (45), (49), and (50).

Proof Because the Gummel map (Theorems 2 and 3) of solving the nonlinear Poisson equation and the continuity equations is continuous, differentiable, and maps the convex set of the $3 M$ tensorated intervals of the bounds onto itself, Brouwer's fixed point theorem guarantees at least one fixed point [18, p. 23].

The uniqueness of the solution for small applied voltages can be shown by repeating the steps in the corresponding section [12]. The decoupling of the Jacobian of the quasiFermi potentials with respect to the electrostatic potential at the thermodynamic equilibrium follows directly from Eq. (40) or the dissipativity of the current relation. The other needed and non-trivial properties of the Jacobian are given in Sect. 9.

\section{Global Newton's method}

The nonlinear equation

$$
\begin{aligned}
\tilde{I}\left(f_{0}, \delta w, v_{l}, v_{r}\right) & =I\left(f_{0}, v_{l}, v_{r}\right)+\delta w=0, \\
f_{0} & =\frac{-j}{J}=\frac{-j \delta x}{\delta w}, \\
I\left(f_{0}, v_{l}, v_{r}\right) & =\int_{v_{l}}^{v_{r}} \frac{f(v) d v}{f(v)-f_{0}}:=\int_{v_{l}}^{v_{r}} i\left(f_{0}, v\right) d v
\end{aligned}
$$

is solved by a local Newton's method, hence the derivative $\frac{\partial I}{\partial f_{0}}$ is known. $\frac{\partial I}{\partial v_{n}}=i\left(f_{0}, v_{n}\right)$ can be computed on the fly after the last function evaluation of the local Newton's method. 
Differentiation of Eq. (52) and application of the chain rule results in all needed derivatives to assemble the Jacobian:

$$
\begin{aligned}
\frac{\partial I}{\partial v_{n}} & =-\frac{\partial I}{\partial f_{0}} \frac{\partial f_{0}}{\partial j} \frac{\partial j}{\partial v_{n}} \\
\frac{\partial j}{\partial v_{n}} & =-i\left(f_{0}, v_{n}\right) /\left(\frac{\partial I}{\partial f_{0}} \frac{\partial f_{0}}{\partial j}\right), n=l, r,
\end{aligned}
$$

while the derivative with respect to $\delta w$ reads as

$$
\begin{aligned}
\frac{\partial I}{\partial f_{0}} \frac{\partial f_{0}}{\partial \delta w}+1 & =-\frac{\partial I}{\partial f_{0}} \frac{\partial f_{0}}{\partial j} \frac{\partial j}{\partial \delta w}, \\
\frac{\partial j}{\partial \delta w} & =-\left(\frac{\partial I}{\partial f_{0}} \frac{\partial f_{0}}{\partial \delta w}+1\right) /\left(\frac{\partial I}{\partial f_{0}} \frac{\partial f_{0}}{\partial j}\right),
\end{aligned}
$$

Using $\frac{\partial f_{0}}{\partial \delta w}=\frac{j \delta x}{(\delta w)^{2}}=-\frac{f_{0}}{\delta w}, \frac{\partial f_{0}}{\partial j}=\frac{-1}{J}$ completes the post-processing of the local Newton's method data. Transformation of the expression to the combination of potentials used is possible, as well.

\section{Properties of the Jacobian}

Knowing the solution $f_{0}$ of the integral equation (39) and the existence of an intermediate value $\tilde{v}$ (see Eq. (40)), is sufficient to derive the qualitative properties of the Jacobian. It is sufficient to show this for one edge. Further, Eq. (40) provides the simplest way to derive a new initial guess for solving the integral equation.

Theorem 5 The Jacobian with respect to the electrostatic potential $w_{l}, w_{r}$ is a symmetric negative semidefinite matrix for each edge. Because one assumes $\Delta w_{i}$, the initial guess $(i=0)$, and each solution of Newton's method at step $i$ to fulfill homogeneous Dirichlet boundary conditions, the global matrix is symmetric negative definite.

Proof Using Eq. (57) and differentiation of equation (18) with respect to $f_{0}$ results in

$$
\frac{\partial I}{\partial f_{0}}=\frac{I}{f(\tilde{v})-f_{0}}=-\frac{\delta w}{f(\tilde{v})-f_{0}},
$$

hence

$$
\left(\frac{\partial j}{\partial w_{l}}, \frac{\partial j}{\partial w_{r}}\right)=-\tilde{G} \frac{f(\tilde{v})}{\delta x}, \tilde{G}=(-1,1),
$$

hence $\tilde{G}^{T} \gamma \tilde{G} \frac{f(\tilde{v})}{\delta x}$ reads as

$$
A_{v_{p}, w_{e d g e}}=\left(\begin{array}{rr}
a & -a \\
-a & a
\end{array}\right), \quad a:=-\gamma \frac{f(\tilde{v})}{\delta x}<0
$$

Because the domain is assumed to be connected or each subdomain has a nonvanishing Dirichlet measure, related columns have a positive row sum.

Theorem 6 Using the above assumptions of Theorem 4 on the grid and the boundary conditions, the Jacobian with respect to the chemical potential of the holes $v_{l}$ and $v_{r}$ is a weakly diagonally dominant M-matrix.

Proof Direct calculation yields for one edge

$\begin{aligned} b_{l} & :=\frac{\partial I}{\partial v_{l}}=-\frac{\left(f(\tilde{v})-f_{0}\right) f\left(v_{l}\right) \gamma}{\left(f\left(v_{l}\right)-f_{0}\right) \delta x}<0, \\ b_{r} & :=\frac{\partial I}{\partial v_{r}}=\frac{\left(f(\tilde{v})-f_{0}\right) f\left(v_{r}\right) \gamma}{\left(f\left(v_{r}\right)-f_{0}\right) \delta x}>0,\end{aligned}$

hence

$A_{v_{p}, v_{p_{e d g e}}}=\tilde{G}^{T}\left(b_{l}, b_{r}\right)=\left(\begin{array}{rr}-b_{l} & -b_{r} \\ b_{l} & b_{r}\end{array}\right)$

The other derivatives are diagonal matrices of the form $V \mathcal{F}^{\prime}, V\left(R^{\prime}(1-r)-R r^{\prime}\right)$.

Remark 3 The use of high order or less local discretizations may result in a loss of definiteness of the Jacobi matrices $A_{v_{p}, w_{\text {edge }}}, A_{v_{n}, w_{\text {edge }}}$, which are well defined elliptic operators in the analytic case, and a coupling of the continuity and the electrostatic potential equation in the thermodynamic equilibrium, compare Eq. (35).

If using discretization schemes that are not unconditionally stable, estimating the smallness conditions for guaranteed definiteness of these matrices results in a characterization of the 'safe to apply area' of these discretizations. With respect to the properties of the Schur complement of $A_{w, w}$ lost definiteness is equivalent to negative densities.

Schemes that result in coupled, linearized continuity equations and the electrostatic potential equation in the thermodynamic equilibrium can realize multiple thermodynamic equilibrium solutions or spurious bifurcation very close to the equilibrium.

\section{Approximation of special functions}

As the Fermi-Dirac integrals show, for typical functions $f(v), \mathcal{F}(v)$ one should expect at least one intermediate region between two asymptotic branches. Here the situation is considered where for $\lim _{v \rightarrow-\infty}$ an exponential behavior and for $\lim _{v \rightarrow \infty}$ rational powers dominate. If one is interested in using analytic integration one faces restrictions for $f(v)$. On the other hand, the benefit is an approximation of the transport up to rounding errors. 


\subsection{Extension of the Blakemore approximation}

The Blakemore approximation [19]

$\mathcal{F}_{1 / 2}(x) \approx \frac{1}{e^{-x}+0.27}$

can be used for $x<1$ and is easily generalized and covers the left asymptotic, hence one looks in general for functions $f_{B g}$ of the form

$f_{B g}(x)=\frac{P_{n}(x)}{P_{m}(x)+e^{-\frac{Q_{k}(x)}{Q_{l}(x)}}}$

or similar ones, where $P_{i}, Q_{i}$ denote polynomials of degree $i$. One needs $\lim _{x \rightarrow-\infty}$ of $\frac{Q_{k}(x)}{Q_{l}(x)}=-x$ and $\frac{P_{n}(x)}{P_{m}(x)}=1$, hence $k=l+1, m=n$ follows.

Checking the integral tables [20] restricts (59) to

$$
f_{B_{3}}(x)=\frac{1 / c}{b / c+e^{-a x}}=\frac{1}{b+c e^{-a x}} .
$$

With $a=1, c=1$ one can extend the asymptotic expression from the leftmost approximation interval. Unfortunately this is only a three parameter approximation, hence one can not have a Hermite spline approximation. On the other hand, it is easy to prove: for functions with a decaying, positive logarithmic derivative a unique solution exists for the parameters $a, b, c$ if $f\left(v_{a}\right), f^{\prime}\left(v_{a}\right)$, (or $\left.f^{\prime}\left(v_{b}\right)\right)$ and $f\left(v_{b}\right)$ are specified.

\subsection{Nonlinear equations for the edge currents}

The Blakemore approximation Eq. (60) on intervals yields

$$
\hat{b}=b-\frac{1}{f_{0}}, f_{B}(v)=b+c e^{-a v}
$$

with $f_{B}$ as the nominator of $f_{B_{3}}$ (the interval indices and sums are omitted, all functions are continuous but not necessarily the integrals-due to discontinuous individual coefficients as weights, hence here the formulas are written in differences per interval)

$$
\begin{aligned}
I_{B_{3}}: & =\int \frac{f(v) d v}{f(v)-f_{0}} \approx \frac{-1}{f_{0}} \int \frac{d v}{\frac{-1}{f_{0}}+f_{B}(v)}=-\delta w, \\
& =\left.\frac{-1}{f_{0}}\left(\frac{a v}{a \hat{b}}-\frac{1}{-a \hat{b}} \ln \left(\hat{b}+c e^{-a v}\right)\right)\right|_{v_{a}} ^{v_{b}} \\
& =\left.\frac{-1}{f_{0} a \hat{b}}\left(\ln e^{a v}+\ln \left(\hat{b}+c e^{-a v}\right)\right)\right|_{v_{a}} ^{v_{b}} \\
& =\left.\frac{-1}{a f_{0} \hat{b}} \ln \left(\hat{b} e^{a v}+c\right)\right|_{v_{a}} ^{v_{b}}=\frac{-1}{a f_{0} \hat{b}} \ln \left(\frac{\hat{b} e^{a v_{b}}+c}{\hat{b} e^{a v_{a}}+c}\right) .
\end{aligned}
$$

Using $I_{B_{3}}=-\zeta_{i} \delta w$ for multiple intervals $i$ each contribution to the sum over the piecewise approximation can be transformed into a Bernoulli function $\mathrm{b}(s)=s /\left(e^{s}-1\right)$ :

$e^{\zeta \delta w f_{0} a \hat{b}}=\frac{\hat{b} e^{a v_{b}}+c}{\hat{b} e^{a v_{a}}+c}$.

Using $z:=\zeta \delta w f_{0} a$,

$$
\begin{aligned}
e^{z \hat{b}}-1 & =\frac{-\hat{b}}{c}\left(e^{a v_{a}} e^{z \hat{b}}-e^{a v_{b}}\right), \\
z & =\frac{z \hat{b}}{c}\left(\frac{-e^{a v_{a}} e^{z \hat{b}}}{e^{z \hat{b}}-1}+\frac{e^{a v_{b}}}{e^{z \hat{b}}-1}\right), \\
z c & =\mathrm{b}(z \hat{b}) e^{a v_{b}}-\mathrm{b}(-z \hat{b}) e^{a v_{a}},
\end{aligned}
$$

and $z \hat{b}=\left(\zeta \delta w f_{0} a\right)\left(b-\frac{1}{f_{0}}\right)=j \zeta \delta x a b-\zeta \delta w a$ yields

$-j \zeta \delta x a c=\mathrm{b}(-z \hat{b}) e^{a v_{b}}-\mathrm{b}(z \hat{b}) e^{a v_{a}}$.

This is a set of fixed point equations for $\zeta_{i} j$ and

$$
\sum_{i=n}^{m} \zeta_{i}=1
$$

determines the Lagrange parameter $j$. The start and end interval of the integration are denoted by $n$ and $m$. This dependency of the decomposition is not present in the sum (or the related product) in Eq. (61). Equation (62) is very well suited to solve the problem for $\delta v \approx 0, v_{r}<v_{B}$, and one interval. Due to $\mathrm{b}^{\prime}(s)+\mathrm{b}^{\prime}(-s)=-1$, the fixed point equation converges for $\left|b / c e^{a \bar{v}}(1+a|\delta v| / 2)\right|<1$, while the derivative with respect to $j$ of the Newton's method related function of Eq. (62) is negative, due to $\mathrm{b}^{\prime}(s)<0$ for finite potentials. Hence the implicit function theorem results in one solution.

All approximation parameters can be expressed by the approximated function, a detailed discussion is omitted. For $a=1, b=0, c=1$, and one interval $(\zeta=1)$ the Eq. (62) reproduces the classic Scharfetter-Gummel scheme.

The Blakemore type approximations are well suited to represent the close to exponential branch (and the saturation in case of the Fermi-Dirac integral of index -1). The case $m=n$ does not fit the large argument asympthotics well and introduces severe approximation problems that translate into refinement requirements, compare Fig. 1.

\subsection{Padé spline approximation}

The right asymptotic requires a Padé type approximation due to $\lim _{x \rightarrow \infty} F_{i}(x)=x^{i+1}, F_{i}(x)$ Fermi-Dirac integral of index $i$. In case $i=j / k>-1$ one would look for approximations of the form 


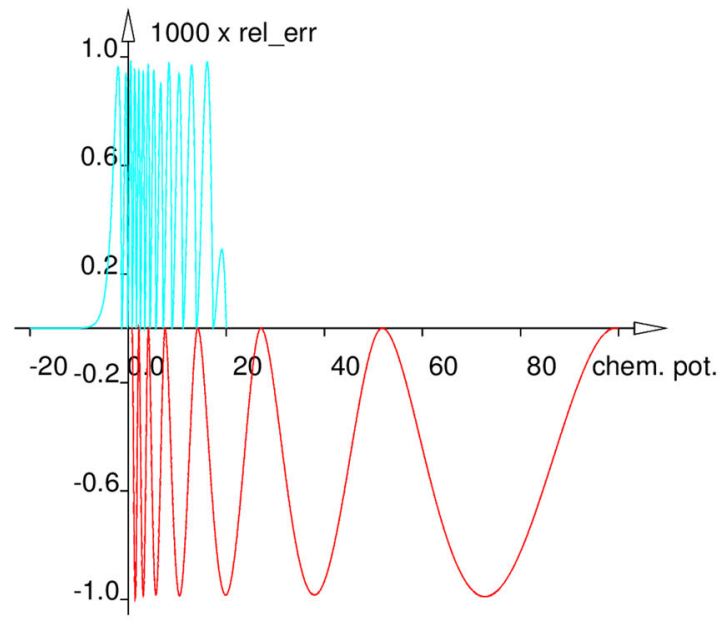

Fig. 1 Typical approximation properties of a Blakemore type approximation with three parameters (blue, above the $\mathrm{x}$ axes) and Padé splines $\left(F_{1 / 2}(v)\right.$ with relative errors $1 / 1000$ and $2.5 / 100$, larger errors violate

$$
f_{P}(x)=\frac{P_{n}(x)}{Q_{m}(x)}
$$

with

$$
\begin{aligned}
P_{n}(x) & =\sum_{i} p_{i} x^{i}+p_{n_{i}(j)} x^{n_{i}(j)}, \\
Q_{m}(x) & =\sum_{i} q_{i} x^{i}+q_{n_{i}(k)} x^{n_{i}(k)},
\end{aligned}
$$

and $n_{i}(j)$ denotes selected multiples of $j$ to force the approximation of the asymptotic part. The intermediate range is addressed by the small powers $x^{i}$. In case of analytic integration the choice is

$P_{1,2}(x)=\frac{a_{0}+a_{1} x}{b_{0}+b_{1} x+b_{2} x^{2}}, a_{0}=1$,

which, by chance, has the wanted asymptotic for $f(x)=$ $F_{1 / 2}^{\prime}(x)$. This is essential for transport in $\mathbb{R}^{3}$ and does not require the computation of a $f_{0}$-dependent partial fraction decomposition. Specifying function and first derivative at $v_{a}$ and $v_{b}$ results in a Hermite spline that provides a convex/concave approximation of convex/concave functions $f(v)$, respectively.

It holds

$$
\begin{aligned}
I & -\left(x_{i+1}-x_{i}\right)=\int_{x_{i}}^{x_{i+1}} \frac{f_{0} d x}{f(x)-f_{0}} \\
& \approx \int_{x_{i}}^{x_{i+1}} \frac{f_{0}\left(b_{0}+b_{1} x+b_{2} x^{2}\right) d x}{a_{0}+a_{1} x-f_{0}\left(b_{0}+b_{1} x+b_{2} x^{2}\right)} \\
& =-\int_{x_{i}}^{x_{i}+1} \frac{\left(b_{0}+b_{1} x+b_{2} x^{2}\right) d x}{b_{0}-a_{0} / f_{0}+\left(b_{1}-a_{1} / f_{0}\right) x+b_{2} x^{2}}:=\tilde{I}\left(f_{0}\right) .
\end{aligned}
$$

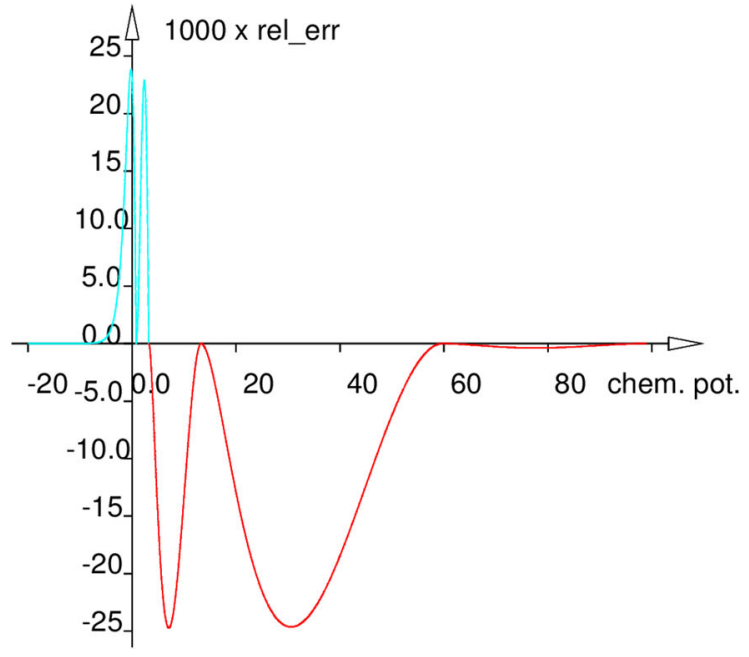

the monotonicity of the approximation, red, dark, below the $\mathrm{x}$ axes). At collocation points the error is zero (Color figure online)

The denominator notation in the last line is changed to

$$
\begin{aligned}
p(x) & :=c_{2} x^{2}+c_{1} x+c_{0}, \Delta=4 c_{2} c_{0}-c_{1}^{2}, \\
c_{0} & =b_{0}-a_{0} / f_{0}, c_{1}=b_{1}-a_{1} / f_{0}, c_{2}=b_{2},
\end{aligned}
$$

where $\Delta$ is the discriminate. Under the assumption $\Delta \neq 0$, hence excluding $\int \frac{d x}{\left(x-x_{0}\right)^{2}}$, the elementary integrals are $(\Delta>$ $0, \Delta<0)$ :

$$
\begin{gathered}
\tilde{I}_{0}:=\int \frac{d x}{c_{2} x^{2}+c_{1} x+c_{0}}=\left\{\begin{array}{r}
\frac{2}{\sqrt{\Delta}} \arctan \frac{2 c_{2} x+c_{1}}{\sqrt{\Delta}} \\
\frac{1}{\sqrt{-\Delta}} \ln \left|\frac{2 c_{2} x+c_{1}-\sqrt{-\Delta}}{2 c_{2} x+c_{1}+\sqrt{-\Delta}}\right|
\end{array}\right. \\
\int \frac{x d x}{p(x)}=\frac{1}{2 c_{2}} \ln |p(x)|-\frac{c_{1}}{2 c_{2}} \tilde{I}_{0}, \\
\int \frac{x^{2} d x}{p(x)}=\frac{x}{c_{2}}-\frac{c_{1}}{2 c_{2}^{2}} \ln |p(x)|+\frac{c_{1}^{2}-2 c_{2} c_{0}}{2 c_{2}^{2}} \tilde{I}_{0},
\end{gathered}
$$

hence

$$
\begin{aligned}
I= & \left(1-\frac{b_{2}}{c_{2}}\right) x-\left(\frac{b_{1}}{2 c_{2}}-\frac{c_{1} b_{2}}{2 c_{2}^{2}}\right) \ln |p(x)|- \\
& \times\left(b_{0}-\frac{c_{1}}{2 c_{2}} b_{1}+\frac{c_{1}^{2}-2 c_{2} c_{0}}{2 c_{2}^{2}} b_{2}\right) \tilde{I}_{0},
\end{aligned}
$$

where $c_{2}=b_{2}$, hence in the coefficients of the spline (up to $\left.p, \tilde{I}_{0}\right)$

$I=-\frac{1}{2 b_{2}}\left(a_{1} / f_{0} \ln |p(x)|+\left(2 b_{2} a_{0}-b_{1} a_{1}+a_{1}^{2} / f_{0}\right) \tilde{I}_{0} / f_{0}\right)$.

To handle the cases $\delta v \approx 0, v_{B}<v_{l}$, and $\Delta \rightarrow 0$ for $f_{0} \rightarrow f_{0}^{*}$, an expansion at $x_{i}\left(\right.$ or $x_{i+1}, \ldots$ ) with respect to $\Delta$ 
using $r=2 c_{2} x+c_{1}, d=\sqrt{ \pm \Delta}$ and the derivatives $r^{\prime}, d^{\prime}$, $r=r\left(x, f_{0}\right), d=d\left(f_{0}\right)$ yields

$$
\begin{aligned}
& \left(\frac{2}{d} \arctan \frac{r}{d}\right)^{\prime}=\frac{-2 d^{\prime}}{d^{2}} \arctan \frac{r}{d}+\frac{2}{d} \frac{d^{2}}{d^{2}+r^{2}}\left(\frac{r^{\prime} d-r d^{\prime}}{d^{2}}\right), \\
& \times\left(\frac{1}{d} \ln \left|\frac{r-d}{r+d}\right|\right)^{\prime}=\frac{-d^{\prime}}{d^{2}} \ln \left|\frac{r-d}{r+d}\right|+\left(\frac{1}{d}|\cdot|^{\prime} \frac{2\left(r^{\prime} d-d^{\prime} r\right)}{(r+d)(r-d)}\right) .
\end{aligned}
$$

$|\cdot|^{\prime}=\operatorname{sign}(\cdot)$ does not cause any problems for arguments close to 1 .

Hence, for prefactors independent of $d$ (or common in both integrals) and $d \rightarrow 0\left(\left|\frac{d}{r}\right|<<1\right)$ holds

$$
\begin{aligned}
& \frac{2}{d}\left(\arctan \frac{r_{l}+\left(r_{r}-r_{l}\right)}{d}-\arctan \frac{r_{l}}{d}\right) \approx \\
& \frac{2}{d} \frac{d^{2}}{d^{2}+r_{l}^{2}} \frac{r_{r}-r_{l}}{d}=\frac{2\left(r_{r}-r_{l}\right)}{d^{2}+r_{l}^{2}}, \\
& \frac{1}{d} \ln \left|\frac{r_{r}-d}{r_{r}+d} \cdot \frac{r_{l}+d}{r_{l}-d}\right|=\frac{1}{d} \ln \left|\frac{1+\left(r_{r}-r_{l}\right) /\left(r_{l}-d\right)}{1+\left(r_{r}-r_{l}\right) /\left(r_{l}+d\right)}\right| \approx \\
& \frac{1}{d} \ln 1+\frac{r_{r}-r_{l}}{r_{l}-d}-\frac{r_{r}-r_{l}}{r_{l}+d} \approx \frac{2\left(r_{r}-r_{l}\right)}{r_{l}^{2}-d^{2}} .
\end{aligned}
$$

The integrals are continuous for $d \rightarrow 0, r_{l}>0$, and $\frac{-c_{1}}{2 c_{2}} \notin\left[x_{i}, x_{i+1}\right]$.

Figure 1 shows the relative error of both approximations. One could try to glue Eqs. (59) and (64) together. That would not be helpful for this application: in case of analytic integration the constraints are very large and numerical integration would be based on sets of precomputed values anyway.

\subsection{Numerical solution}

Theorem 1 provides the basis for the numerical solution. The first step is to deduce the sign and size relation of $\delta v$ and $\delta w$, hence to select the actual case (i), (ii), or (iii). The discussion of the special cases, rough numerical integration, the edge current of the last global step of Newton's method or a predictor, ... or those cases, where Eq. (25) can be used, provide an initial guess for $f_{0}$ together with bounds. Hence, with two (in some cases three) function evaluations one sign change of $h\left(f_{0}\right):=I\left(f_{0}\right)+\delta w=0$ can be constructed, starting with the initial guess. Some care is necessary to handle the rapid and slow variation of $h$, depending on the distance $\left|f_{0, k}-f_{0}\right|$ whit the iteration index $k$. It is useful to keep a short history of left and right arguments and function values for computing the new estimate and a save switching decision to Newton's method, if $h$ and $h^{\prime}$ are consistent with the history. The case small $|\delta v|$ can typically be restricted to approximation of data using one interval only.

Figure 2 compares edge currents for the classical Scharfetter-Gummel scheme, an approximation of $F_{1 / 2}$ with relative errors smaller $1 \%$, and the classical Blakemore approximation with arguments $\left(\varphi_{l}, \varphi_{r}, w_{l}\right)$ in the cube
$[-5,5]^{3}$. As expected, overestimated saturation effects show up in the classical Blakemore approximation.

\section{Discussion and summary}

Mean value theorem based arguments are sufficient to show essential stability properties for discrete drift-diffusion problems and a general construction principle of spatial discretizations together with boundary conforming Delaunay grids. The proposed construction results in a unique, dissipative solution of an integral equation defining the edge current and reproducing the dissipation of the one dimensional problem up approximation errors of the governing function, which can be made smaller than the typical errors in the data of the problem. This generalized ScharfetterGummel scheme is compatible with the thermodynamic equilibrium and the characteristic structure of the Jacobian of the analytic problem. A detailed discussion for distribution functions of special interest in different semiconductor device applications supplements the general facts. The short hint to variational formulations should help to understand the implications of the variational formulation for the structure of special models. On the other hand, the assumption that the velocity is the gradient of a potential can be relaxed by either introducing a potential on a local scale or proper discretizations of a constant velocity onto edges.

If the strict monotonicity of the density with respect to the chemical potential is lost, the so called charge-neutral approximation does not exist for sufficiently large doping values and, for instance, large applied voltages at gate boundaries. Before that happens one is entering the problems associated with phase separation and degenerate diffusion in case of a variational formulation. If $f(v)$ is not anymore strictly monotone, the Cauchy problem may have more than one solution for the given boundary data. Dissipativity would still be a selection criterion. Formally, the integration can be performed by the same technique: for instance one mirrors $f(v)$ at its maximum at $v_{m} \in\left[v_{l}, v_{r}\right]$, hence it is a monotone function and a correction of the known difference of the integral is possible using $\left(f\left(v_{m}\right)-f(\infty)\right)\left(v_{r}-v_{m}\right)$. The essential problem is to prove bounds for the solution of the analytic problem away from $\min (\mathcal{F}(v)), \max (\mathcal{F}(v))$ or to study the consequences of degenerate diffusion together with the interaction potential defined by the Poisson equation.

The intuition based on physics would suggest two (or more) types of particles, each described by monotone functions (or convex free energy functionals) and one ore more reactions, that relate the densities in the proper way.

For models of organic (amorphous) semiconductors, like EGDM [4], it would be interesting to find states close to degenerate diffusion either by numerical solution of the com- 


\section{(a) Boltzmann}

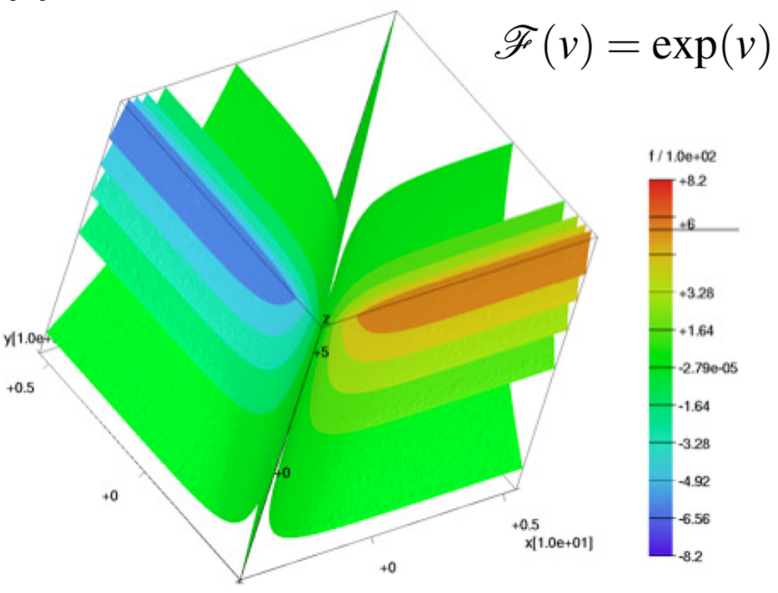

(b) Fermi-Dirac

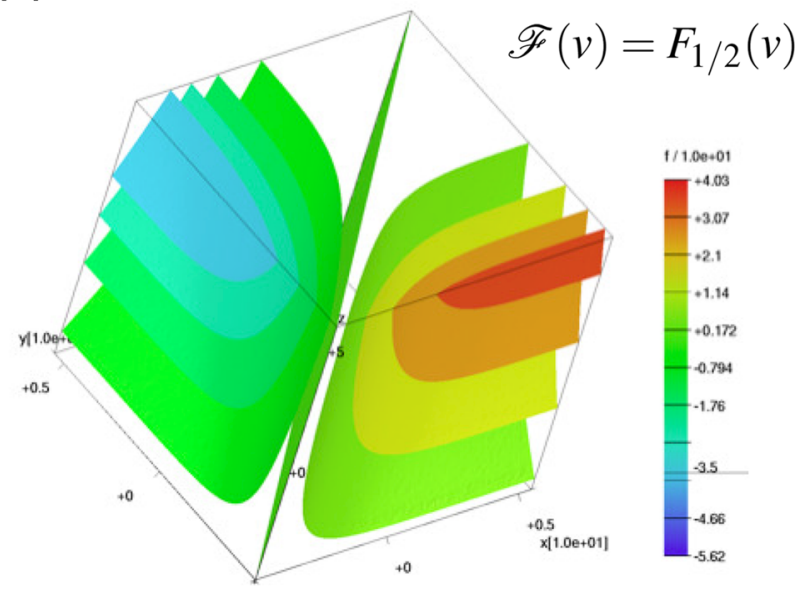

(c) Blakemore approximation

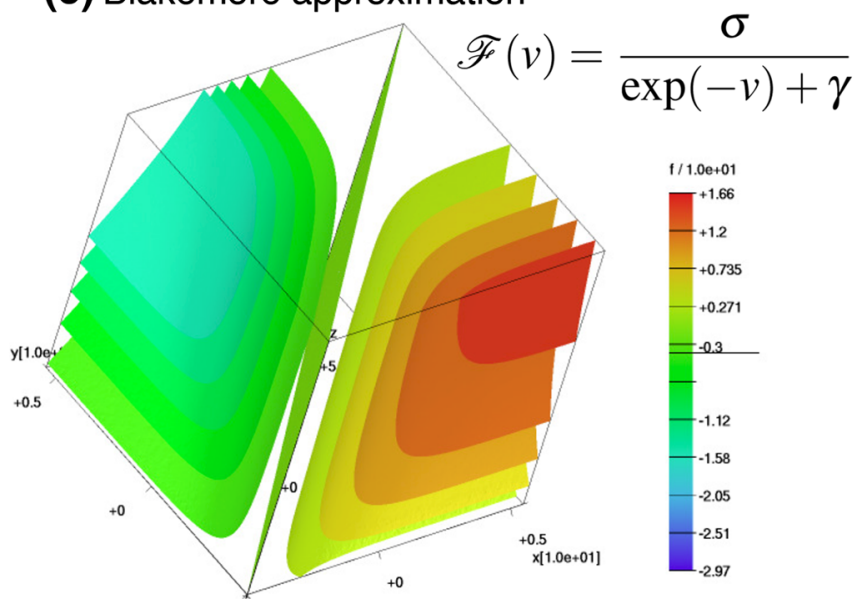

Fig. 2 Influence of the distribution function on the edge current: shown are isosurfaces of the current for $10^{6}$ random argument triples (in $U_{T}$ ) for the Boltzmann case (a), the Fermi-Dirac integral $F_{1 / 2}$ (b), and the original Blakemore approximation $(\sigma=1, \gamma=0.27$, c.). The ampli-

plete set of model equations or experimentally. To some extent (solution methods are expected to deteriorate) model changes with respect to $f(v), \mathcal{F}(v)$ are reduced to changing approximating functions.

Acknowledgments The author thanks A. Glitzky and J. A. Griepentrog for very helpful discussions, H. Doan for the close to rounding error Polylog data used for the approximations, and T. Koprucki for the pointer to [19]. The reviewer comments are acknowledged, too. Their more outside point of view was very helpful to improve the readability.

\section{References}

1. Van Roosbroeck, W.: Theory of flow of electrons and holes in germanium and other semiconductors. Bell Syst. Tech. J. 29, 560607 (1950)

2. Scharfetter, D.L.: Large-signal analysis of a silicon read diode oscillator. IEEE Trans. Electr. Dev 16, 64-77 (1969) tude varies by one order of magnitude. $x, y, z$ correspond to $\varphi_{a}, \varphi_{b}, w_{a}$; $w_{b}=0$. The correction terms for nonvanishing drift break the symmetry with respect to interchanging $\varphi_{a}$ and $\varphi_{b}$, present in the Boltzmann case

3. Becker, Julian, Gärtner, Klaus, Klanner, Robert, Richter, Rainer: Simulation and experimental study of plasma effects in planar silicon sensors. Nucl. Instrum. Methods Phys. Res. Sect. A 624(3), 716-727 (2010)

4. Pasveer, W.F., et al.: Unified description of charge-carrier mobilities in disordered semiconducting polymers. Phys. Rev. Lett. 94, 206601 (2005)

5. Gajewski, H., Gärtner, K.: A dissipative discretization scheme for a nonlocal phase segregation model. Z. Angew. Math. Mech. 85, 815-822 (2005)

6. Gajewski, H., Gröger, K.: Semiconductor equations for variable mobilities based on Boltzmann statistics or Fermi-Dirac statistics. Math. Nachr. 140, 7-36 (1989)

7. Gajewski, H., Gärtner, K.: On the discretization of van Roosbroeck's equations with magnetic field. Z. Angew. Math. Mech. 76, 247-264 (1996)

8. Gajewski, H., Gröger, K.: Reaction-diffusion processes of electrically charged species. Math. Nachr. 177, 109-130 (1996)

9. Gajewski, H., Albinus, G., Hünlich, R.: Thermodynamic design of energy models of semiconductor devices. Nonlinearity $\mathbf{1 5}, 367-$ 383 (2002) 
10. Glitzky, A., Gärtner, K.: Existence of bounded steady state solutions to spin-polarized drift-diffusion systems. SIAM J. Math. Anal. 41, 2489-2513 (2010)

11. Glitzky, A., Gärtner, K.: Energy estimates for continuous and discretized electro-reaction-diffusion systems. Nonlinear Anal. 70, 788-805 (2009)

12. Gärtner, K.: Existence of bounded discrete steady-state solutions of the van Roosbroeck system on boundary conforming delaunay grids. SIAM J. Sci. Comput. 31(2), 1347-1362 (2009)

13. Griepentrog, J.A.: On regularity, positivity and long-time behavior of solutions to an evolution system of nonlocally interacting particles. Report, WIAS, Preprint No. 1932 (2014)

14. Liero, M., Mielke, A.: Gradient structures and geodesic convexity for reaction-diffusion systems. Phil. Trans. R. Soc. A 371, 20120346 (2013). (28 pages)
15. Bonč-Bruevič, V.L., Kalashnikov, S.G.: Halbleiterphysik. Deutscher Verlag der Wissenschaften, Berlin (1982)

16. Delaunay, B.: Sur La Sphére Vide. Izvestia Akademii Nauk SSSR. Otd. Matem. i Estestv. Nauk 7, 793-800 (1934)

17. Si, H.: Three dimensional boundary conforming delaunay mesh generation. PhD thesis, TU Berlin (2008)

18. Zeidler, E.: Vorlesung über nichtlineare Funktionalanalysis IFixpunktsätze. Teubner, Leipzig (1976). in German

19. Blakemore, J.S.: Approximations for Fermi-Dirac integrals, especially the function $\mathcal{F}_{1 / 2}(\eta)$ used to describe electron density in a semiconductor. Solid State Electron. 25, 1067-1076 (1982)

20. Gradstein, I.S., Ryzhik, I.M.: Tables of Integrals, Sums, Series, and Products. Fizmatgiz, Moskow (1962). in Russian 